\title{
Synthesis and photophysics of 6-dimethylaminopurine-capped Q-CdS nanoparticles-a study of its photocatalytic behavior
}

\author{
Anil Kumar ${ }^{\dagger}$ and Shipra Mital \\ Department of Chemistry, Indian Institute of Technology Roorkee, Roorkee 247667, India
}

\begin{abstract}
Nanoparticles of CdS have been stabilized in aqueous medium by surface capping with 6dimethylaminopurine at $\mathrm{pH} 11.0$ in the presence of excess $\mathrm{Cd}^{2+}$. The nucleation of the cluster is controlled through coordination of 6-dimethylaminopurine via. $-\mathrm{N}\left(\mathrm{CH}_{3}\right)_{2}$ group. Excess 6-dimethylaminopurine binds to the core structure through H-bonding involving protonated N(9). CdS capped with 6-dimethylaminopurine produced relatively small sized particles $(2 \mathrm{~nm})$ having a narrow size distribution. These particles did not exhibit any Ostwald's ripening and its solid sample could be fully redispersed in aqueous medium. Thermolysis results in the growth of these particles but demonstrates a better photocatalytic activity. Relaxation kinetics reveals the surface to contain a large number of surface states and the emission is originated by the presence of sulfur vacancies on the particle. The addition of indole simply quenches the emission of CdS in a bimolecular collisional process at a diffusion-controlled rate of about $9 \times 10^{9} \mathrm{dm}^{3} \mathrm{~mol}^{-1} \mathrm{~s}^{-1}$. Irradiation of the reaction mixture containing CdS and indole by light of $\lambda>360 \mathrm{~nm}$ results in the formation of indigo with a quantum efficiency of 0.1 .
\end{abstract}

\section{INTRODUCTION}

During the last two decades there has been a tremendous advancement in the colloids chemistry involving metals and semiconductors, which has led to the development of a new branch, the nanoscience and nanotechnology [1-9]. The modification of the surface of nanoparticles plays an important role in manipulating their physicochemical, photophysical and photocatalytic properties [5-8]. Recently, highly luminescent CdSe nanocrystals capped with CdS [10], TOPO and ZnS [11] have been reported. The capping of inorganic semiconductor nanocrystals by biological molecules has been explored extensively for their stabilization, to produce them with narrow size distribution, for enhancing their solubility in aqueous medium and to exploit them as sensors for biological systems [12-19]. The stability in these clusters might be controlled by the particle-particle interaction through hydrogen bonding. Such systems are also helpful in the manipulation of their photostability. Lately, purine and adenine-capped Q-CdS have been investigated in order to analyze the role of interfaces in polynucleotide-capped CdS particles [20, 21]. The electronic and photocatalytic properties of these materials are influenced by the nature of surface-capping agent. In these experiments, purine was found to control the size of the cluster in a dynamic equilibrium. Purinecapped Q-CdS crystallites demonstrated relatively broad-size distribution having large average particle size but depicted high photocatalytic activity [20] better than even coupled $\mathrm{Cd}(\mathrm{OH})_{2}$-coated Q-CdS-TiO

\footnotetext{
†E-mail: anilkfcy@iitr.ernet.in
}

particles [22]. On the other hand adenine-capped Q-CdS demonstrated a better control for the manipulation of electronic properties but exhibited relatively poor photoactivity [21]. In case of adenine the electronic properties are controlled by its interaction through amino group with the particle [23]. In the present work we have synthesized 6-dimethylaminopurinecapped Q-CdS and examined its photophysical and photocatalytic properties. As regards to improve their electronic properties, 6-dimethylaminopurine may be considered as a better capping agent than adenine, because $-\mathrm{N}\left(\mathrm{CH}_{3}\right)_{2}$ group in 6-dimethylaminopurine is a better electron donating group compared to that of $-\mathrm{NH}_{2}$ in adenine. The present work will allow us to verify the above findings with other previously investigated purines.

\section{MATERIALS AND METHODS}

2.1. Reagents. Cadmium perchlorate, adenine, 6dimethylaminopurine (6-DMAP), indole, 3-methylindole (Aldrich); purine, indole-3-ethanol, dl-tryptophan (sigma); sodium hydroxide, perchloric acid (Merck); hydrochloric acid (Qualigens); $\mathrm{N}_{2}$ (purity > 99.9\%) were of analytical grade and were used as received without further purification.

2.2. Equipment. Electronic spectra were measured on a Shimadzu UV-2100S spectrophotometer. Fluorescence spectra were recorded on a Shimadzu RF-5301PC spectrofluorophotometer. The size and size distribution of the particles were determined on a Philips CM-10 transmission electron microscope. Water from the colloidal solution of CdS was removed on a Buchi $\mathrm{R}-114$ Rotavapor at $35^{\circ} \mathrm{C}$. The resulting solid sample 

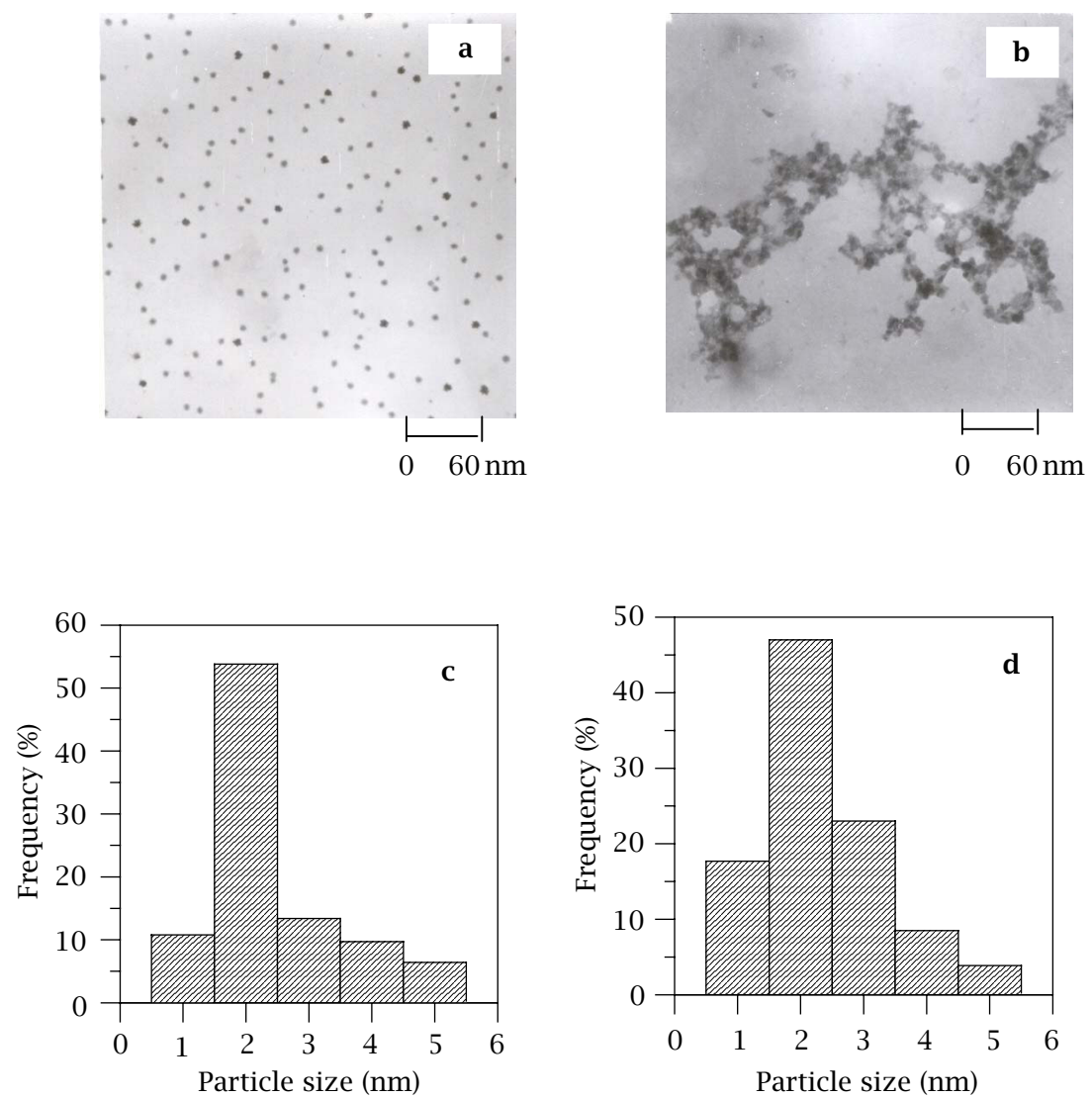

Figure 1. Electron micrograph(s) and size histogram(s) of 6-dimethylaminopurine-capped Q-CdS containing $5 \times$ $10^{-3} \mathrm{~mol} \mathrm{dm}^{-3}$ observed at different locations $(\mathrm{a} ; \mathrm{c})$ and $(\mathrm{b} ; \mathrm{d})$.

of CdS was used for its characterization by XRD, IR and NMR spectroscopy. X-ray powder diffraction patterns were recorded on a Philips APD-10 X-ray diffractometer with the $\mathrm{Cu} \mathrm{K}_{\alpha}$ line of the X-ray source. The acceleration voltage was set at $40 \mathrm{kV}$ with $30 \mathrm{~mA}$ flux. IR spectra were recorded on a Perkin-Elmer "Spectrum-2000" IR spectrometer in far and mid IR ranges using polyethylene and $\mathrm{KBr}$ media, respectively. Proton NMR spectra were measured on a Bruker DPX-300 MHz spectrometer in DMSO using $\mathrm{Si}\left(\mathrm{CH}_{3}\right)_{4}$ as an internal reference. The fluorescence lifetime in picosecond/nanosecond time domain were measured on an IBH-5000U fluorescence spectrometer using a picosecond Tsunami Ti-sapphire mode locked laser as excitation source. Hamamatsu photomultiplier was used for the detection of fluorescence. Decay curves were kinetically analyzed by using a multi-exponential fitting program provided by IBH. The goodness-of-fit was judged by computing $x^{2}$ from a plot of weighed residuals and auto-correlation function. Continuous photolysis was performed on an Oriel Photolysis assembly equipped with $200 \mathrm{~W} \mathrm{Hg}$ (Xe) arc lamp.

2.3. Methodology. 6-DMAP-capped Q-CdS was synthesized by using the previously reported method [20,

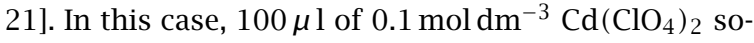
lution was injected into $100 \mathrm{ml}$ of aqueous solution of 6-DMAP containing its varied concentrations. The $\mathrm{pH}$ of this solution was maintained at 11.0. Q-CdS was prepared by injecting $5 \times 10^{-5} \mathrm{~mol} \mathrm{dm}^{-3}$ of $\mathrm{SH}^{-}$to the degassed mixture. The appearance of a faint yellow color was consistent with the formation of CdS.

The size of the particles was determined by applying a small drop of colloidal CdS solution on a carbon coated copper G-200 grid (size $3.05 \mathrm{~mm}$ ). The electron micrographs along with their histograms for a typical $5 \times 10^{-3} \mathrm{~mol} \mathrm{dm}^{-3}$ of 6-DMAP-capped Q-CdS observed at different locations of the same grid are shown in Figure 1 . These particles exhibit relatively a narrow size distribution ranging from 1 to $5 \mathrm{~nm}$ with an average size of about $2 \mathrm{~nm}$. A decrease in the concentration of 6-DMAP resulted in an increase in the size of the particle and the size distribution became broad.

For the investigation of the characteristic behavior of these particles Q-CdS capped with $5 \times 10^{-3} \mathrm{~mol} \mathrm{dm}^{-3}$ of 6-DMAP was used. Steady-state fluorescence spectra were corrected for the background/instrument response. Lifetime measurements were made by using excitation light of $375 \mathrm{~nm}$ and emission was measured at different wavelengths. The reactant and product(s) 
from the irradiated reaction mixture were extracted in chloroform.

\section{RESULTS AND DISCUSSION}

\subsection{Electronic properties of 6-DMAP-capped Q- CdS}

\subsubsection{Optical properties}

Figure 2 presents the electronic spectra of 6-DMAPcapped Q-CdS $\left(5 \times 10^{-5} \mathrm{~mol} \mathrm{dm}^{-3}\right)$ at $\mathrm{pH} 11.0$ in the presence of varied concentrations of 6-DMAP. An increase in concentration of 6 -DMAP from $5 \times 10^{-4}$ to $5 \times$ $10^{-3} \mathrm{~mol} \mathrm{dm}{ }^{-3}$ causes the onset of absorption to shift from 2.61 to $2.92 \mathrm{eV}$, respectively. At $5 \times 10^{-3} \mathrm{~mol} \mathrm{dm}^{-3}$, the excitonic peak is fairly blue shifted and becomes more prominent. Although, at all used concentrations of 6-DMAP, CdS particles could be stabilized for a fairly long period but the highest stability was obtained with particles having 6-DMAP concentrations of $5 \times 10^{-3} \mathrm{~mol} \mathrm{dm}^{-3}$. This oxygenated colloidal solution, stored in a stoppered vessel, was found to remain stable for more than 12 and 14 months at room temperature and $5{ }^{\circ} \mathrm{C}$, respectively. At low $\mathrm{pH}$ these colloidal solutions were relatively unstable. At pH 8.0 the colloidal solution of CdS containing a typical $5 \times 10^{-3} \mathrm{~mol} \mathrm{dm}^{-3}$ of 6-DMAP coagulated in about $30 \mathrm{~min}$. At this $\mathrm{pH}$ the excitonic peak, observed at pH 11.0 (Figure 2), vanished completely.

The stabilization of CdS particles by 6-DMAP might occur due to interaction of $\mathrm{Cd}^{2+}$ through $\mathrm{N}(7), \mathrm{N}(9)$ or $-\mathrm{N}\left(\mathrm{CH}_{3}\right)_{2}$. The electronic spectrum of 6-DMAP in the absence and presence of $\mathrm{Cd}^{2+}$ is shown in Inset: Figure 2. As the spectrum of 6-DMAP virtually remains unchanged in the presence of $\mathrm{Cd}^{2+}$, it suggests the absence of any electronic interaction of $\mathrm{Cd}^{2+}$ with 6-DMAP in 6-DMAP capped Q-CdS.

\subsubsection{Emission behavior}

Figure 3 shows that at low concentration of 6-DMAP the emission spectrum is very broad having emission peaks centered at 580 and $650 \mathrm{~nm}$. An increase in the concentration of 6-DMAP blue shifts the $580 \mathrm{~nm}$ emission gradually with an increase in its intensity but the emission intensity of red band is reduced. A change in concentration from $5 \times 10^{-4}$ to $5 \times 10^{-3} \mathrm{~mol} \mathrm{dm}^{-3}$ blue shifts the band-gap emission from 2.14 to $2.36 \mathrm{eV}$. The direction of shift in emission spectra are very similar to that observed in the optical spectra and exhibits the size quantization effect. A further increase in the concentration of 6-DMAP, however, did not exhibit any further change in emission behavior. Moreover, at this concentration a white turbidity appeared within few hours, which is likely to have occurred due to the poor solubility of 6-DMAP. For the oxygenated solution of 6-DMAPcapped Q-CdS the quantum efficiency of fluorescence

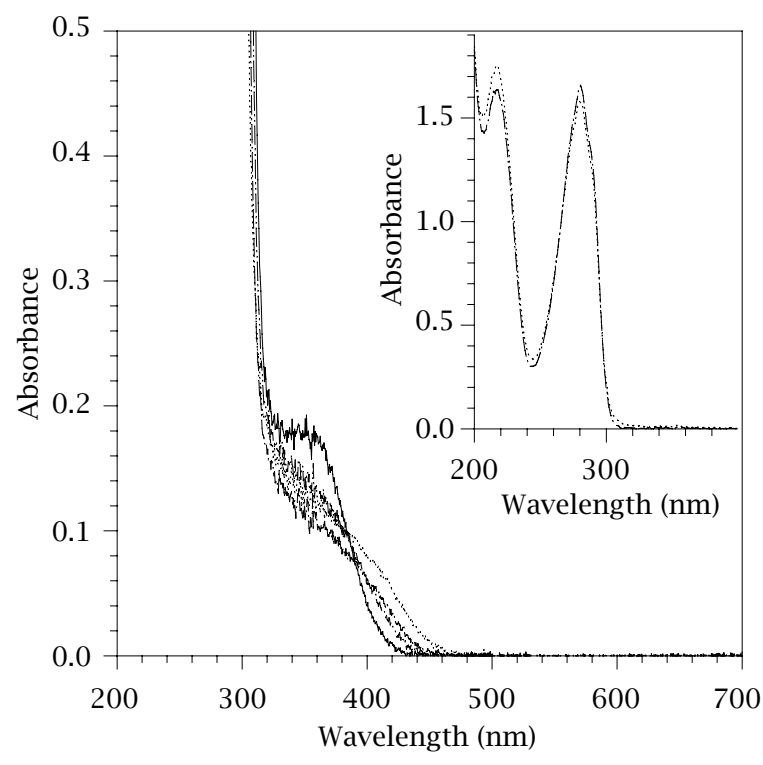

Figure 2. Electronic spectra of 6-dimethylaminopurinecapped Q-CdS $\left(5 \times 10^{-5} \mathrm{~mol} \mathrm{dm}^{-3}\right)$ at $\mathrm{pH} 11.0$ in the presence of different amounts of 6-dimethylaminopurine $\left(\times 10^{-3} \mathrm{~mol} \mathrm{dm}{ }^{-3}\right)-0.5$ (. . . ) 1.0 (-.-); 2.0 (-..-); 5.0 (-) added prior to precipitation of CdS. Inset: Electronic spectra of 6-dimethylaminopurine $\left(5 \times 10^{-4} \mathrm{~mol} \mathrm{dm}^{-3}\right)$ in the $a b$ sence $(\cdots)$ and presence of $\mathrm{Cd}^{2+}\left(1.0 \times 10^{-4} \mathrm{~mol} \mathrm{dm}^{-3}\right)$ (-.-) at pH 11.0.

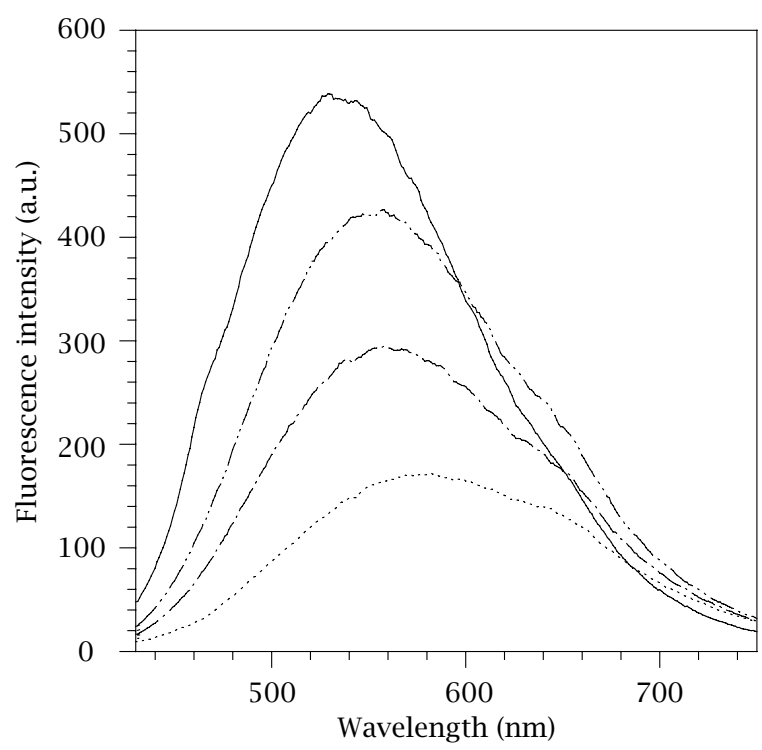

Figure 3. Emission spectra of 6-dimethylaminopurinecapped Q-CdS $\left(5 \times 10^{-5} \mathrm{~mol} \mathrm{dm}^{-3}\right)$ at $\mathrm{pH} 11.0$ in the presence of different amounts of 6-dimethylaminopurine $\left(\times 10^{-3} \mathrm{~mol} \mathrm{dm}^{-3}\right)-0.5(\cdots)$ ); $1.0(-.-)$; 2.0 (-..-); 5.0 (-) added prior to precipitation of CdS. $\lambda_{\mathrm{ex}}=400 \mathrm{~nm}$.

$\left(\phi_{\mathrm{f}}\right)$ at $525 \mathrm{~nm}$ was estimated to be 0.03 . In the absence of $\mathrm{O}_{2}$ the $\phi_{\mathrm{f}}$ increased to about 0.05. A decrease in the 
Table 1. Comparision of observed d values in XRD pattern of 6-dimethylaminopurine-capped Q-CdS with that of standard values for Cubic-CdS, Hexagonal-CdS and $\mathrm{Cd}(\mathrm{OH})_{2}$.

\begin{tabular}{cccc}
\hline Observed Value & Cubic-CdS & Hexagonal-CdS & $\mathrm{Cd}(\mathrm{OH})_{2}$ \\
\hline 3.59 & - & 3.58 & - \\
3.36 & 3.36 & 3.37 & - \\
3.16 & - & 3.16 & - \\
2.88 & - & - & 2.91 \\
2.40 & - & - & 2.41 \\
2.07 & 2.06 & - & - \\
1.61 & 1.75 & - & - \\
\hline
\end{tabular}

concentration of 6-DMAP below $5 \times 10^{-4} \mathrm{~mol} \mathrm{dm}^{-3}$ stabilized these particles poorly, as the solution became turbid within $30 \mathrm{~min}$. At pH 8.0, the emission maximum of 6 -DMAP $\left(5 \times 10^{-3} \mathrm{~mol} \mathrm{dm}^{-3}\right)$-capped Q-CdS was found to red shift to $545 \mathrm{~nm}$ and the intensity of emission is reduced by a factor of four.

\subsubsection{X-ray diffraction}

The X-ray diffraction patterns of powder 6-DMAPcapped Q-CdS were recorded by scanning in $2 \theta$ range of 20 to $70^{\circ}$. The observed d-spacing indicates the 6DMAP-capped Q-CdS is produced in both cubic and wurtzite crystal structural forms (Table 1) by comparing them with the literature value [24]. It also exhibits the presence of $\mathrm{Cd}(\mathrm{OH})_{2}$ in this mixture.

\subsubsection{IR spectra}

IR spectra of $\mathrm{Cd}(\mathrm{OH})_{2}$-coated Q-CdS, $\mathrm{Cd}^{2+}-6$-DMAP complex and 6-DMAP-capped Q-CdS powders were recorded in both far and mid IR region. In literature, bulk CdS is known to depict a broad absorption in 350 to $100 \mathrm{~cm}^{-1}$ [25]. 6-DMAP-capped Q-CdS also shows a broad band along with several peaks centered on it in this wavenumber range. The presence of additional peaks on the broad band clearly indicates the interaction of CdS with 6-DMAP as observed earlier with other purines [20, 21]. In mid IR spectrum the $-\mathrm{N}$ $\mathrm{CH}_{3}$ stretching in 6-DMAP-capped Q-CdS shifts to lower wave number side to $3387 \mathrm{~cm}^{-1}$ from $3424 \mathrm{~cm}^{-1}$ in basic 6-DMAP and becomes fairly broad. It clearly indicates a strong interaction of $\mathrm{CdS}$ through $-\mathrm{N}\left(\mathrm{CH}_{3}\right)_{2}$. Thus IR spectra recorded in both far and mid IR suggest a chemical interaction of 6-DMAP with Q-CdS.

\subsubsection{NMR spectra}

In the proton NMR spectrum of free basic 6-DMAP, the pyrimidine, imidazole and methyl protons were observed at $8.04,7.82,3.42$, and $2.50 \mathrm{ppm}$, respectively. The values corresponding to these protons for 6 DMAP-capped Q-CdS were observed at 8.02, 7.77, 3.42, and $2.51 \mathrm{ppm}$, respectively. The values belonging to pyrimidine and imidazole protons are slightly shifted to upfield whereas the protons of the methyl group attached to nitrogen virtually remains unchanged. In a similar experiment with adenine-capped Q-CdS, the protons belonging to amino group exhibit relatively a large downfield shift [26]. The downfield shift suggests the deshielding of $-\mathrm{NH}_{2}$ protons due to interaction of $-\mathrm{NH}_{2}$ group with $\mathrm{Cd}^{2+}$ of $\mathrm{CdS}$ through lone pair of electrons. Such interaction is though expected to enhance through $-\mathrm{N}\left(\mathrm{CH}_{3}\right)_{2}$ group, which would have resulted in larger downfield shift due to protons associated with $\mathrm{N}$ but these protons being present in methyl group are shielded.

\subsubsection{Analysis of origin of emission}

The dynamics of the charge carriers for CdS nanoclusters capped with different amounts of 6-DMAP was followed by monitoring the time-resolved decay of fluorescence. All the decay curves depicted three-exponential decay kinetics. Table 2 presents the lifetime data for all the three components, the average emission lifetime $(\langle\tau\rangle)$ and the $\chi^{2}$ values for these traces at $550 \mathrm{~nm}$. The first two components lie in sub-nanosecond $(0.40 \mathrm{~ns})$ and nanosecond (2.09 ns) time domain and do not show any considerable variation with a change in concentration of 6-DMAP. The component, $\tau_{3}$ had a long time constant (>10 ns), and exhibited a regular increase in the lifetime from 12.28 to $18.00 \mathrm{~ns}$ with an increase in concentration from $1 \times 10^{-3}$ to $5 \times 10^{-3} \mathrm{~mol} \mathrm{dm}^{-3}$ of 6-DMAP, respectively. This component contributed prominently to the emission yield (> 55\%). The fluorescence decay of adenine-capped Q-CdS followed a very similar behavior, except the average lifetime for a variation in concentration from $1 \times 10^{-3}$ to $5 \times$ $10^{-3} \mathrm{~mol} \mathrm{dm}^{-3}$ of adenine used for capping varied from 6.37 to $18.18 \mathrm{~ns}$, respectively. At $5 \times 10^{-3} \mathrm{~mol} \mathrm{dm}^{-3}$ of capping agent $\langle\tau\rangle$ is fairly high for adenine-capped Q-CdS compared to that of 6-DMAP-capped Q-CdS. It suggested the charge carriers are relatively long lived in case of adenine-capped Q-CdS. A reduction in $\langle\tau\rangle$ in case of 6-DMAP-capped Q-CdS might be attributed to the reactivity of hole with 6-DMAP as has been 
Table 2. Effect of [6-dimethylaminopurine] added prior to the precipitation of CdS on the lifetime of CdS at $550 \mathrm{~nm}$.

\begin{tabular}{|c|c|c|c|c|c|c|c|c|}
\hline \multirow{3}{*}{$\begin{array}{l}\text { [6-dimethylaminopurine] } \\
\times 10^{3} \\
\left(\mathrm{~mol} \mathrm{dm}^{-3}\right)\end{array}$} & \multicolumn{7}{|c|}{ Lifetime (ns) } & \multirow{3}{*}{$x^{2}$} \\
\hline & \multicolumn{2}{|c|}{ Component 1} & \multicolumn{2}{|c|}{ Component 2} & \multicolumn{2}{|c|}{ Component 3} & \multirow{2}{*}{$\langle\tau\rangle$} & \\
\hline & $\tau_{1}$ & Emission (\%) & $T_{2}$ & Emission (\%) & $T_{3}$ & Emission (\%) & & \\
\hline \multirow{3}{*}{1.0} & 0.39 & & 1.92 & & 12.28 & & & \\
\hline & $(2.75)$ & 18.36 & $(0.73)$ & 24.38 & $(0.27)$ & 57.26 & 7.57 & 1.05 \\
\hline & 0.42 & & 2.06 & & 14.74 & & & \\
\hline \multirow[t]{2}{*}{2.0} & (3.39) & 15.94 & $(0.99)$ & 22.79 & $(0.37)$ & 61.27 & 9.57 & 1.15 \\
\hline & 0.39 & & 1.94 & & 15.23 & & & \\
\hline \multirow[t]{2}{*}{3.5} & $(3.71)$ & 12.56 & $(1.21)$ & 20.53 & $(0.50)$ & 66.90 & 10.63 & 1.19 \\
\hline & 0.39 & & 1.98 & & 18.00 & & & \\
\hline 5.0 & $(4.55)$ & 18.76 & $(0.94)$ & 19.69 & $(0.32)$ & 61.55 & 11.55 & 1.03 \\
\hline
\end{tabular}

*Values given in the brackets denote the pre-exponential factors corresponding to the respective $\tau$

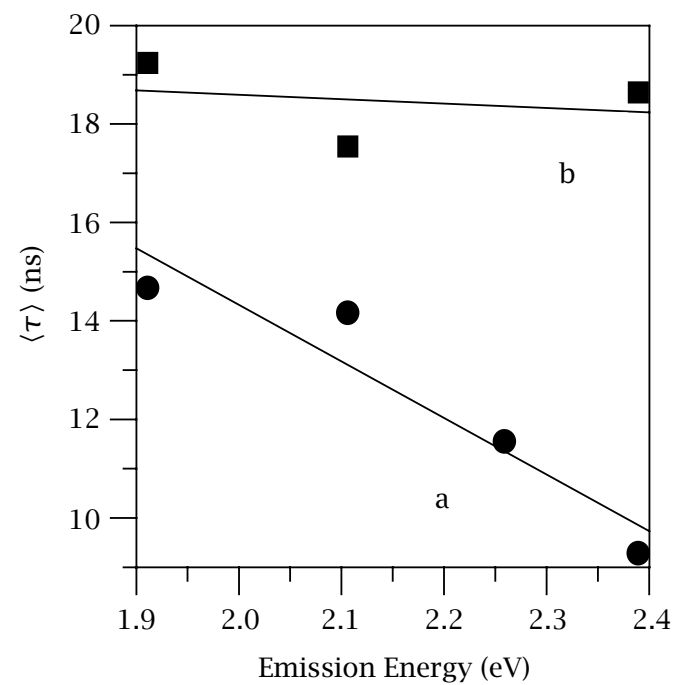

Figure 4. Average lifetime of 6-dimethylaminopurinecapped Q-CdS containing $5.0 \times 10^{-3} \mathrm{~mol} \mathrm{dm}^{-3}$ of 6 dimethylaminopurine at $\mathrm{pH} 11.0$ as a function of the energy of emission before $(\bullet)$ and after thermolysis ( $\mathbf{\square})$. $\lambda_{\mathrm{ex}}=375 \mathrm{~nm}$.

noticed in the steady state fluorescence experiments also. This hypothesis was checked by adding 6-DMAP externally to the colloidal solution of CdS. An addition of $5 \times 10^{-3} \mathrm{~mol} \mathrm{dm}^{-3}$ of 6-DMAP reduced $\langle\tau\rangle$ from 11.55 to $10.14 \mathrm{~ns}$. A plot of $\left\langle\tau_{0}\right\rangle /\langle\tau\rangle v s$. [6-DMAP] follows Stern-Volmer relationship from which the quenching rate constant of $2.5 \times 10^{9} \mathrm{~mol}^{-1} \mathrm{~s}^{-1}$ was evaluated. Had the 6-DMAP not reacted with hole, one could have anticipated the lifetime to be much higher in this case compared to that of adenine-capped Q-CdS.

Different traps, located on the surface of the particles were analyzed by making lifetime measurements as a function of energy of emission (Figure 4, curve a). A decrease in emission energy from 2.39 to $1.91 \mathrm{eV}$ enhanced $\langle\tau\rangle$ considerably from 9.28 to $14.67 \mathrm{~ns}$, respectively. The emission at higher and lower energy originates from shallow and deep traps, respectively [20].

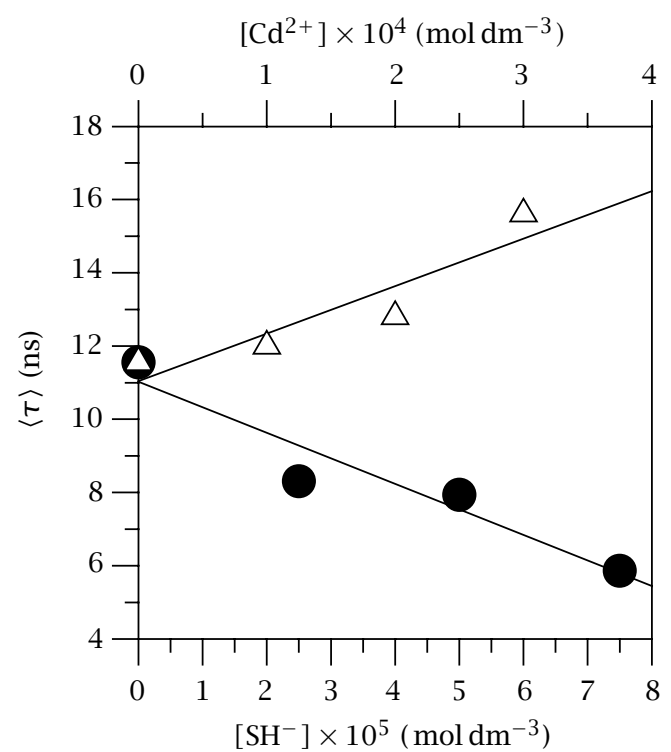

Figure 5. Average lifetime of 6-dimethylaminopurinecapped Q-CdS containing $5.0 \times 10^{-3} \mathrm{~mol} \mathrm{dm}^{-3}$ of 6 dimethylaminopurine at $\mathrm{pH} 11.0$ containing different amounts of $\mathrm{Cd}^{2+}(\triangle)$ and $\mathrm{SH}^{-}(\bullet) . \lambda_{\mathrm{ex}}=375 \mathrm{~nm}$.

The nature of these traps was further examined by the addition of excess $\mathrm{Cd}^{2+}$ and $\mathrm{SH}^{-}$, respectively. A plot of $\langle\tau\rangle v s$. $\left[\mathrm{Cd}^{2+}\right]$ and $\left[\mathrm{SH}^{-}\right]$are shown in Figure 5 . The addition of $\mathrm{Cd}^{2+}$ from $1 \times 10^{-4}$ to $3 \times 10^{-4} \mathrm{~mol} \mathrm{dm}^{-3}$ results in an enhancement of $\langle\tau\rangle$ from 12.00 to $15.61 \mathrm{~ns}$ whereas the addition of $\mathrm{SH}^{-}$from $2.5 \times 10^{-5}$ to $7.5 \times$ $10^{-5} \mathrm{~mol} \mathrm{dm}^{-3}$ reduced the $\langle\tau\rangle$ from 8.30 to $5.86 \mathrm{~ns}$, respectively. Similar behavior of fluorescence decay was observed at other wavelengths.

\subsection{General characteristics}

\subsubsection{Effect of ageing}

The optical and emission behavior of 6-DMAPcapped Q-CdS particles were followed periodically and remained virtually unchanged for about 14 months. 
This feature of these particles is quite different to stoichiometric naked Q-CdS particles prepared using polyphosphate and styrene/maleic anhydride stabilizers, which undergo Ostwald's ripening [27, 28].

\subsubsection{Thermal stability}

Thermolysis of 6-DMAP-capped Q-CdS at $80{ }^{\circ} \mathrm{C}$ for about an hour caused a red shift for the onset and excitonic absorption due to these particles from 2.92 to 2.85 and 3.44 to $3.26 \mathrm{eV}$, respectively. In emission spectrum, it developed a broad emission band at $650 \mathrm{~nm}$. It was also evident by an increase in the particle size. It suggests that these particles behave very similar to those, capped with relatively smaller amount of 6DMAP (Figures 2 and 3). Obviously, upon heating some of the loosely bound 6-DMAP might have fallen into the solution.

For thermolysed particles, a variation in $\langle\tau\rangle$ as a function of emission energy is shown in Figure 4 (curve b). In general, $\langle\tau\rangle$ increased upon thermolysis. This change being the most prominent at higher energy $(2.38 \mathrm{eV})$ where $\langle\tau\rangle$ is increased from 9.28 to $18.64 \mathrm{~ns}$.

\subsubsection{Solubility}

Solid sample of 6-DMAP-capped Q-CdS in the used concentration range could be fully redispersed in aqueous medium. This behavior shows an improvement over purine and adenine-capped Q-CdS, which could be redispersed only partially. The electronic properties and catalytic action of these particles changed in accordance to those observed upon heating the colloidal solution, as water was removed on rotavapor by heating them to $35^{\circ} \mathrm{C}$. This feature is important as regard to use these materials as a reagent in laboratory and to perform the analysis of early events kinetically at their interface.

\subsubsection{Photostability}

The illumination of 6-DMAP-capped Q-CdS by light of $\lambda>360 \mathrm{~nm}\left(1.5 \times 10^{17}\right.$ photons $\left./ \mathrm{s}\right)$ in the presence of oxygen did not bring any noticeable change in its optical characteristics for about $15 \mathrm{~min}$. However, its further irradiation caused an increase in both UV and visible region, which possibly arises due to oxidation of 6-DMAP by photogenerated hole. It is very likely that initial irradiation causes the weakening of the bond of 6-DMAP attached in the outer layer to core 6-DMAP capped Q-CdS, which is eventually oxidized by hole. A decrease in emission intensity of these particles upon irradiation also supports the occurrence of this process. This behavior of 6-DMAP-capped Q-CdS is different to that of adenine-capped Q-CdS where no product of oxidation was detected. It could be understood in terms of the low oxidation potential $(0.78 \mathrm{eV})$ of 6 DMAP compared to that of adenine $(0.82 \mathrm{eV})$ at $\mathrm{pH} 11.0$.

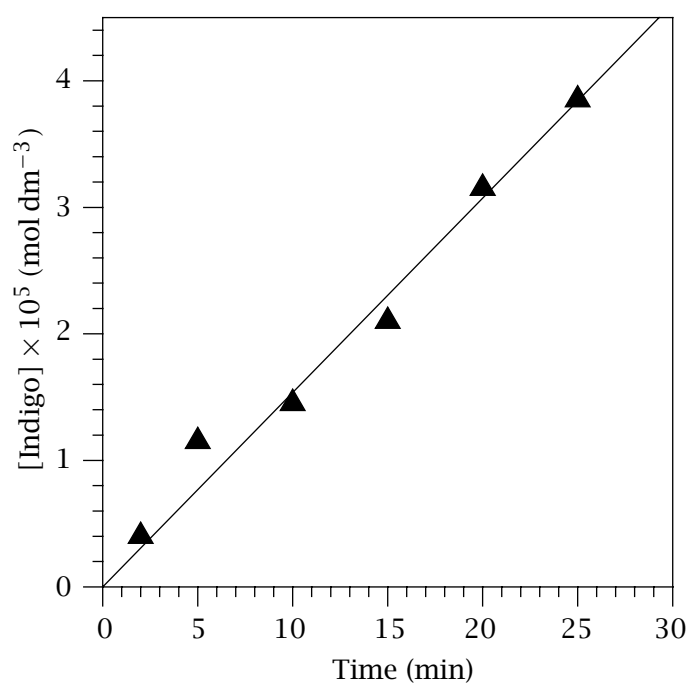

Figure 6. Yield of indigo as a function of illumination time containing indole $\left(1 \times 10^{-2} \mathrm{~mol} \mathrm{dm}^{-3}\right)$ at $\mathrm{pH} 11.0$ using 6dimethylaminopurine-capped $\mathrm{Q}-\mathrm{CdS}(\mathbf{\Lambda})$ as sensitizer.

\subsection{Photocatalytic activity of 6-DMAP-capped Q- CdS particles}

\subsubsection{Photosensitization of indole- $\mathrm{O}_{2}$ redox couple}

The irradiation of reaction mixture containing $5 \times$ $10^{-5} \mathrm{~mol} \mathrm{dm}^{-3}$ 6-DMAP-capped Q-CdS and $1 \times$ $10^{-2} \mathrm{moldm}^{-3}$ of indole at $\mathrm{pH} 11.0$ by light of $\lambda>360 \mathrm{~nm}$, where neither purine nor indole possess any absorption, results in the production of bluish product. It was identified to be indigo by its characteristic absorption spectrum having $\lambda_{\max }$ at $602 \mathrm{~nm}$ and $R_{\mathrm{f}}$ value in TLC experiments using its authentic sample. Its amount in different samples was quantified by measuring the absorption at $602 \mathrm{~nm}$ $\left(\varepsilon=1.74 \times 10^{4} \mathrm{dm}^{3} \mathrm{~mol}^{-1} \mathrm{~s}^{-1}\right)$. The time irradiation of the reaction mixture exhibits a continuous increase in absorption at $602 \mathrm{~nm}$ for about $30 \mathrm{~min}$ from which the quantum efficiency for the formation of indigo was computed to be 0.1 (Figure 6). This value is smaller to that of purine-capped Q-CdS but about three folds higher to that observed with adenine-capped Q-CdS. The sensitization of indole- $\mathrm{O}_{2}$ couple by the thermolysed particles improved the quantum efficiency for the formation of indigo from 0.1 to 0.15 .

The mechanism of this reaction was analyzed by monitoring the photophysics of CdS in the absence and presence of the used redox couple by monitoring steady state and time-resolved fluorescence.

\subsubsection{Photophysics of the particle in the presence of indole}

The addition of indole caused simply the quenching of fluorescence of oxygenated 6-DMAP-capped Q-CdS 
Table 3. Effect of [Indole] on the lifetime of 6-dimethylaminopurine-capped CdS at $550 \mathrm{~nm}$.

\begin{tabular}{|c|c|c|c|c|c|c|c|c|}
\hline \multirow{3}{*}{$\begin{array}{l}{[\text { Indole }] \times 10^{3}} \\
\left(\mathrm{~mol} \mathrm{dm}{ }^{-3}\right)\end{array}$} & \multicolumn{7}{|c|}{ Lifetime (ns) } & \multirow{3}{*}{$x^{2}$} \\
\hline & \multicolumn{2}{|c|}{ Component 1} & \multicolumn{2}{|c|}{ Component 2} & \multicolumn{2}{|c|}{ Component 3} & \multirow{2}{*}{$\langle\tau\rangle$} & \\
\hline & $\tau_{1}$ & Emission (\%) & $\tau_{2}$ & Emission (\%) & $T_{3}$ & Emission (\%) & & \\
\hline \multirow{3}{*}{0} & 0.39 & & 1.99 & & 18.00 & & & \\
\hline & (4.55) & 18.76 & $(0.94)$ & 19.69 & $(0.32)$ & 61.55 & 11.55 & 1.03 \\
\hline & 0.52 & & 2.10 & & 13.89 & & & \\
\hline \multirow[t]{2}{*}{2} & (11.44) & 16.54 & (3.94) & 22.87 & $(1.58)$ & 60.59 & 8.98 & 1.20 \\
\hline & 0.39 & & 1.64 & & 11.86 & & & \\
\hline \multirow[t]{2}{*}{4} & (9.49) & 11.77 & $(4.93)$ & 25.92 & $(1.64)$ & 62.31 & 7.86 & 1.17 \\
\hline & 0.38 & & 1.63 & & 10.65 & & & \\
\hline 10 & $(4.90)$ & 23.96 & $(1.36)$ & 28.45 & $(0.35)$ & 47.59 & 5.62 & 1.01 \\
\hline
\end{tabular}

*Values given in the brackets denote the pre-exponential factors corresponding to the respective $\tau$

without altering its emission maxima. The optical spectrum of CdS also remains unchanged in its presence. The quenching of emission follows Stern-Volmer relationship from which a quenching rate constant of $9.1 \times$ $10^{9} \mathrm{dm}^{3} \mathrm{~mol}^{-1} \mathrm{~s}^{-1}$ was computed. Other indoles like 3methylindole, indole-3-ethanol and dl-tryptophan etc. also depicted similar quenching behavior. It was further verified by measuring the lifetime of CdS in the absence and presence of different concentrations of indole. The lifetime data of these runs are summarized in Table 3 . These data show that in the presence of indole, the components $\tau_{1}$ and $\tau_{2}$ virtually remained unchanged whereas $\tau_{3}$ decreases regularly. Apparently, it is relatively deep among shallow trapped hole, which are involved in the process of quenching. A plot of $\left\langle\tau_{0}\right\rangle /\langle\tau\rangle \nu s$. concentration of indole gives a linear curve from which the quenching rate constant of $8.6 \times 10^{9} \mathrm{dm}^{3} \mathrm{~mol}^{-1} \mathrm{~s}^{-1}$ has been evaluated. This value is very similar to that observed above in steady state experiment (vide supra). These changes in photophysical behavior are very similar to that observed with adeninecapped Q-CdS but differ to that of purine-capped Q-CdS where the interaction of indole demonstrated the formation of an emissive charge transfer intermediate.

6-DMAP stabilizes CdS nanocrystallites nicely as is revealed by the absence of any change in its electronic properties upon ageing. In fact these particles showed better stability in the presence of air than reported earlier with biomolecule capped Q-CdS [19-21]. Increased stability of these colloids is understood by the coordination of 6-DMAP to the particle through $-\mathrm{N}\left(\mathrm{CH}_{3}\right)_{2}$. The possibility of partial complexation through $\mathrm{N}(9)$, however, cannot be ruled out as has been observed with purine. The coordination of particles through $-\mathrm{N}\left(\mathrm{CH}_{3}\right)_{2}$, was however, not evident by NMR spectroscopy which can be explained due to shielding of protons present in $-\mathrm{CH}_{3}$ group. The fact that the higher concentration of 6-DMAP controls the size and size distribution effectively, is a manifestation of the fact that the secondary layer / large amount of 6-DMAP possibly gets bound to its core unit through $-\mathrm{N}(9)$ proton via. $\mathrm{H}$ bonding. It essentially controls the nucleation of these particles and depicts the size quantization effect (Figures 2 and 3). XRD measurements exhibit the presence of CdS in both cubic and hexagonal shapes similar to those observed with purine.<smiles></smiles>

Structure of 6-DMAP-capped Q-CdS

The appearance of sharp excitonic peak in optical absorption and band gap emission with relatively high quantum yield clearly indicate the passivation of the surface of particles by 6-DMAP occurs such that it binds to the shallow traps involved in non-radiative transition. This emission mainly arises from relatively deep among shallowly trapped charge carriers as is revealed by an enhancement of the $\tau_{3}$ component upon increasing the concentration of 6-DMAP, which also contributes largely to the $\phi_{\mathrm{f}}$. The influence on emission behavior and lifetime of the charge carriers upon the addition of excess $\mathrm{Cd}^{2+}$ and $\mathrm{SH}^{-}$clearly suggests these traps to originate mainly due to sulfur vacancies. Under identical conditions of experiments $\langle\tau\rangle$ was higher for 6-DMAP-capped Q-CdS compared to that of purinecapped Q-CdS indicating that 6-DMAP acts as a better 
surface capping agent as regards to manipulate its photophysical behavior. It is also reflected by an increase in the overall quantum efficiency of fluorescence (0.05) compared to that for purine-capped Q-CdS (0.02). The value of average lifetime of charge carriers for 6-DMAPstabilized Q-CdS is, however, smaller compared to that of observed with adenine-stabilized Q-CdS, which is understood by the reactivity of hole with 6-DMAP due to its lower oxidation potential to that of adenine.

An enhancement in lifetime of the charge carriers upon thermolysis with almost similar values at all energy indicates that shallowly lying traps moved to deeper states upon heating and, thereby, did not demonstrate any appreciable variation in the emission lifetime. The enhanced lifetime of the charge carriers also explains the increased photocatalytic activity of the thermolysed particles. Although the quantum efficiency of reactions of indoles using 6-DMAP-capped Q$\mathrm{CdS}$ as sensitizer is about three times higher to that observed using adenine-capped Q-CdS but the mechanism of the catalytic action was similar in the two cases. The reaction of indole in both cases occurred without forming a charge transfer intermediate between $\mathrm{CdS}\left(\mathrm{h}^{+}\right)$ and indole. This behavior is in contrast to that observed with purine-capped Q-CdS where the formation of an emissive exciplex was noted [20]. A better reactivity of hole in the present system compared to that with adenine-capped Q-CdS particles could be rationalized by a difference in the nature of the modified surface by 6 -DMAP. It possibly allows more holes to be made available at the surface through oxidized 6-DMAP to indole(s) like solutes.

In summary 6-DMAP-capped Q-CdS is fairly stable and exhibit better control of size, size-distribution and electronic properties compared to purine and adeninecapped Q-CdS. Further, these particles, being redispersable, can be exploited as a chemical reagent. The mechanism of photocatalytic action of 6-DMAP-capped Q-CdS differed to that of purine-capped Q-CdS but matched to that of adenine-capped Q-CdS. In general physicochemical properties of these particles are very similar to that observed earlier with other purine(s) and verifies those observations.

\section{ACKNOWLEDGMENTS}

We gratefully acknowledge the financial support of DST, New Delhi to undertake the work. SM is thankful to CSIR, New Delhi for the award of SRF. Thanks are also due to Director, NCUFP, Chennai, Prof. M. Dutta, Delhi Univ., Director, AIIMS, New Delhi, Mr. J. Peter, ONGC, Dehradun and Prof. A. S. Brar, IIT Delhi for providing us the facilities of Single Photon counter, FTIR, TEM, XRD and NMR, respectively.

\section{REFERENCES}

[1] A. Henglein, Top. Curr. Chem. 143 (1988), 115.

[2] G. Schmid, Chem. Rev. 92 (1992), 1709.

[3] P. V. Kamat, J. Phys. Chem. B 106 (2002), 7729.

[4] A. Henglein, Langmuir 15 (1999), 6738.

[5] A. P. Alivisatos, J. Phys. Chem. B 100 (1996), 13226.

[6] A. Eychmüller, J. Phys. Chem. B 104 (2000), 6514.

[7] J. Z. Zhang, J. Phys. Chem. B 104 (2000), 7239.

[8] H. Weller, Adv. Mat. 5 (1993), 88.

[9] P. V. Kamat, Chem. Rev. 93 (1993), 267.

[10] X. Peng, M. C. Schlamp, V. Kadavanich, and A. P. Alivisatos, J. Am. Chem. Soc. 119 (1997), 7019.

[11] K. Hashizume, M. Matsubayashi, M. Vacha, and T. Tani, J. Lumin. 98 (2002), 49.

[12] C. T. Dameron, R. N. Reese, R. K. Mehra, A. R. Kortan, P. J. Carroll, M. L. Steigerwald, L. E. Brus, and D. R. Winge, Nature 338 (1989), 596.

[13] M. Bruchez Jr., M. Moronne, P. Gin, S. Weiss, and A. P. Alivisatos, Sci. 281 (1998), 2013.

[14] L.-Y. Wang, L. Wang, F. Gao, Z.-Y. Yu, and Z.-M. Wu, Analyst 281 (2002), 2013.

[15] M. C. Brelle, J. Z. Zhang, L. Nguyen, and R. K. Mehra, J. Phys. Chem. A 103 (1999), 10194.

[16] P. R. Smith, J. D. Holmes, D. J. Richardson, D. A. Russell, and J. R. Sodeau, J. Chem. Soc. Faraday Trans. 94 (1998), 1235.

[17] M. Nirmal, B. O. Dabbousi, M. G. Bawendi, J. J. Macklin, J. K. Trautman, T. D. Harris, and L. E. Brus, Nature 383 (1996), 802.

[18] L. Xin, and J. L. Coffer, Chem. Mat. 11 (1999), 2326.

[19] J. L. Coffer, S. R. Bigham, R. F. Pinizzotto, and H. Yang, Nanotechnol. 3 (1992), 69.

[20] A. Kumar and S. Mital, Photochem. Photobiol. Sci. 1 (2002), 737.

[21] A. Kumar and S. Mital, J. Colloid Interface Sci. 240 (2001), 459.

[22] A. Kumar and A. K. Jain, J. Mol. Catal. A: Chem. 165 (2001), 265.

[23] A. Kumar and S. Mital, J. Colloid Interface Sci. 265 (2003), 432.

[24] W. F. McClune, Powder Diffraction File Search Manual for Frequently Encountered Phases, Pennsylvania, USA, 1978.

[25] R. A. Nyquist and R. O. Kagel, Infrared Spectra of Inorganic Compounds, Academic Press, New York, 1971.

[26] The proton NMR spectrum of adenine-capped QCdS exhibited a downfield shift of $-\mathrm{NH}_{2}$ protons to 4.25 ppm compared to that of basic adenine, which was observed at $3.76 \mathrm{ppm}$.

[27] A. Fojtik, H. Weller, U. Koch, and A. Henglein, Ber. Bunsenges. Phys. Chem. 88 (1984), 969.

[28] R. Rossetti, J. L. Ellison, J. M. Gibson, and L. E. Brus, J. Chem. Phys. 80 (1984), 4464. 


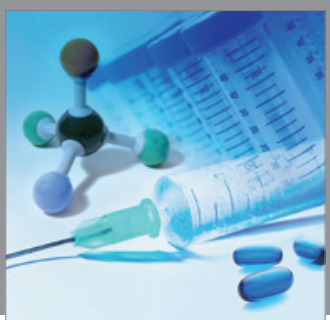

International Journal of

Medicinal Chemistry

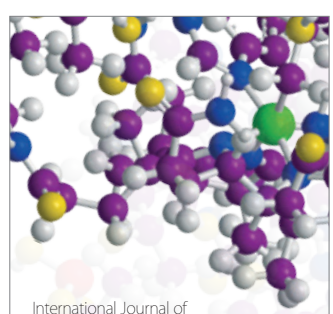

Carbohydrate Chemistry

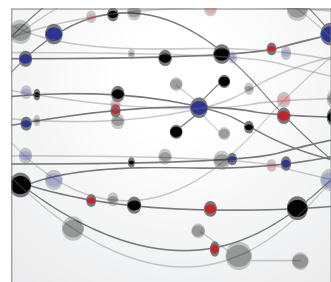

The Scientific World Journal
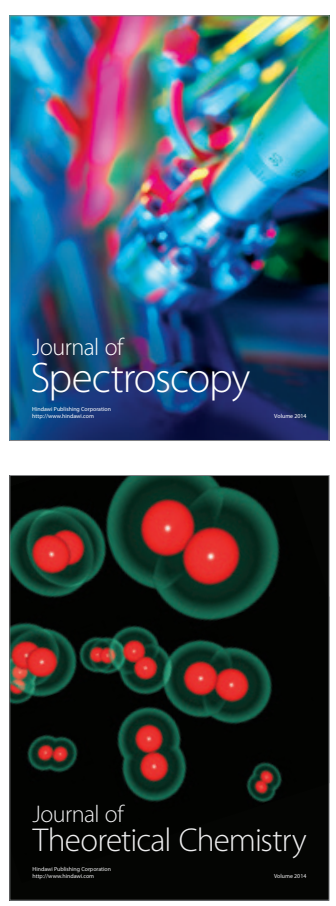
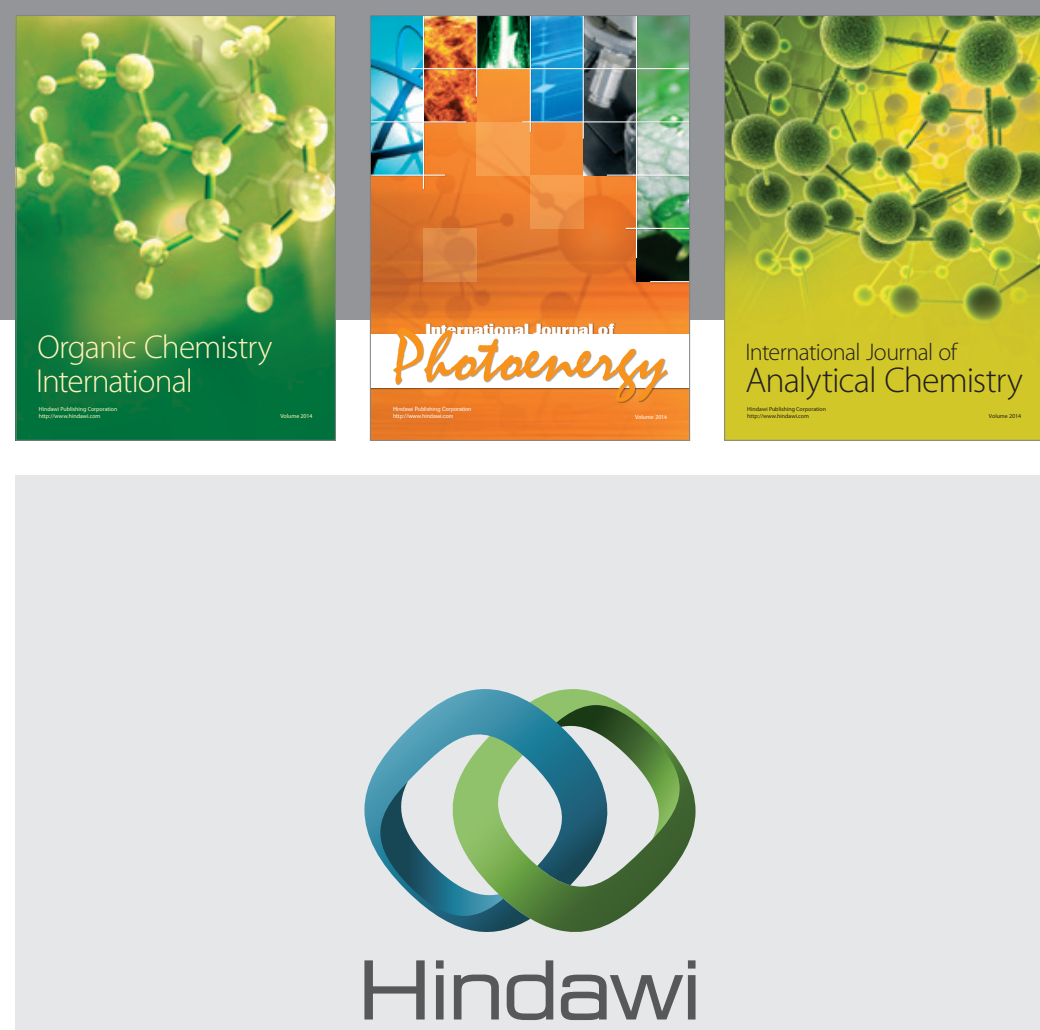

Submit your manuscripts at

http://www.hindawi.com
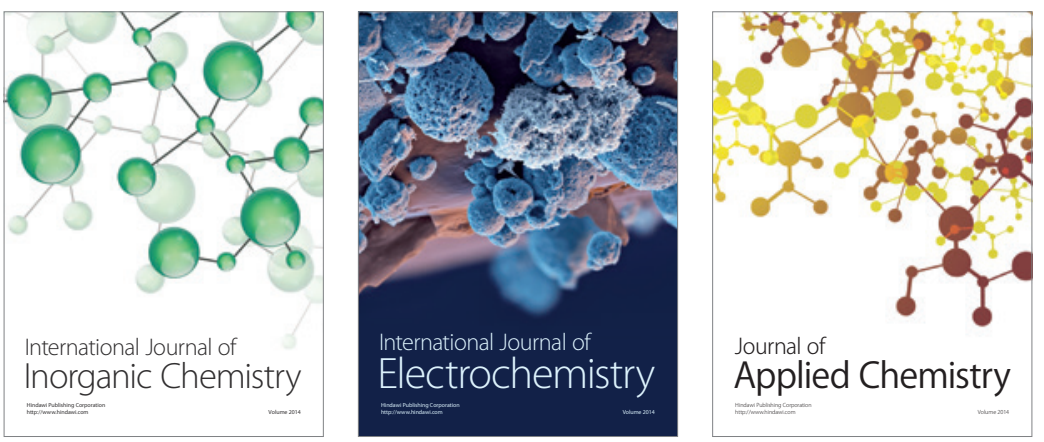

Journal of

Applied Chemistry
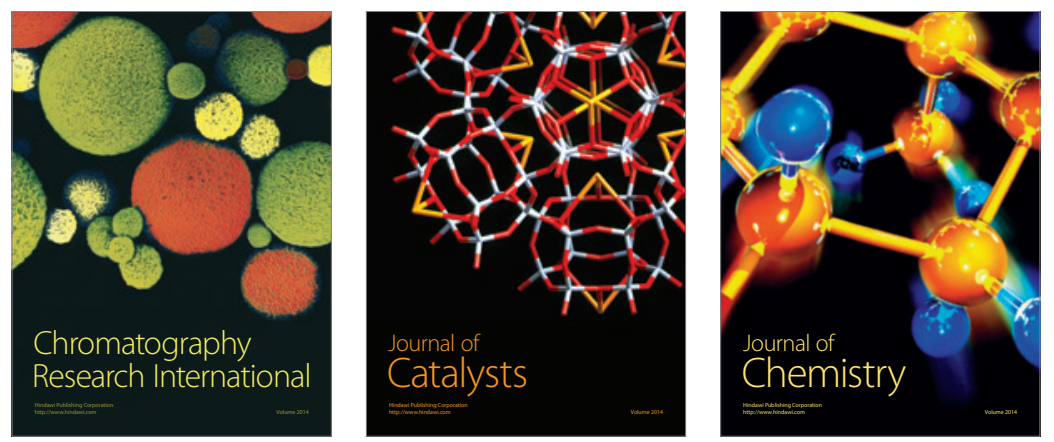
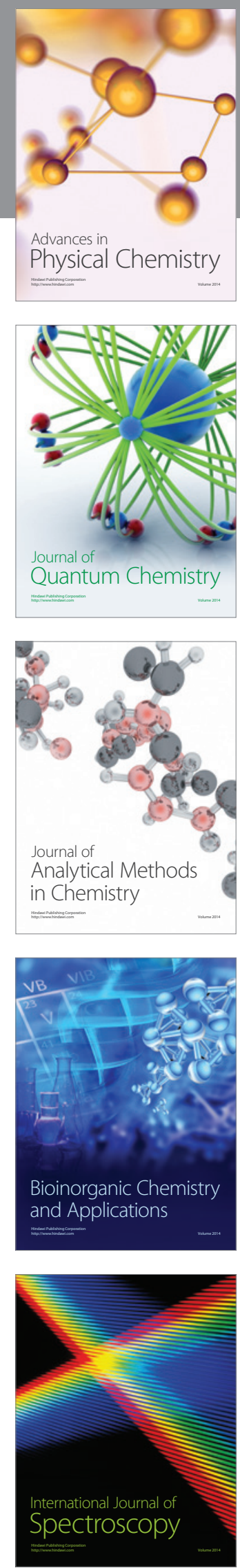